\title{
Automatic Detection of Exudates Using Morphological Approach
}

\author{
Pankaj Bachani, Dhaval Patel, Ravi Parikh \\ E \& C Department, Parul Institute of Engineering And Technology PIET-Limda, Vadodara,India
}

\begin{abstract}
Diabetic retinopathy (DR) is a sight threatening complication due to diabetes mellitus that affects the retina .The blindness due to DR can be prevented if detected early enough for treatment.Hence screening for early stage of $D R$ is very important. An ophthalmologist uses an ophthalmoscope to visualize the blood vessels and his or her brain to detect the DR stages. The above pathology-based method is time consuming and often requires fluorescein angiograms for accurate diagnosis. It requires highly trained and skilled clinicians to perform the DR severity grading method.

In this paper we have proposed a low cost retinal diagnosis system which can aid an ophthalmologist to quickly diagnose various stages of diabetic retinopathies.

Keywords:Diabetic retinopathy, diagnosis Ophthalmologist,fluorescein angiograms.
\end{abstract}

\section{Introduction}

Diabetes Mellitus (DM) is the name of a chronic, systemic, life-threatening disease. It occurs when the pancreas does not secrete enough insulin or the body is unable to process it properly. This results in an abnormal increase in the glucose level in the blood. Over time this high level of glucose causes damage to blood vessels. This damage affects both eyes and nervous system, as well as heart, kidneys and other organs.

In general there are two types of diabetes. Diabetes type 1 results from a failure of the human body to produce insulin. Type 1 diabetes is less common than type 2 diabetes. People with type 1 diabetes take insulin injections.

It is estimated that $90-95 \%$ of Americans, who are diagnosed with diabetes, have type 2 diabetes . This form of diabetes usually develops in adults age 40 and older and is most common in the age group over age 55 . About $80 \%$ of people with type 2 diabetes are overweight. It was reported that type 2 diabetes is often part of a metabolic syndrome that includes obesity, elevated blood pressure, and high levels of blood lipids.

\section{Causes}

The recent increase in diabetes can be attributed to an aging population and increasing prevalence of obesity as well as sedentary life habits. Genetic inheritance plays a role in both, type 1 and type 2 diabetes. But it appears that type 1 diabetes is also triggered by some (mainly viral) infections. There is also a genetic element in individual susceptibility to some of these triggers which has been traced to particular human leukocyte antigen genotypes. However, even in those who have inherited the susceptibility, type $1 \mathrm{DM}$ seems to require an environmental trigger. Some evidence indicates that the B4 virus might be such a trigger.

\section{Effects} affects briefly.

Diabetes affects the kidney, eyes, nerves and heart. In the following sections, we have discussed these

\section{Diabetic Nephropathy}

Diabetic nephropathy is the main cause of end-stage renal diseases. When the body digests protein it contaminates the blood with waste products. The kidneys filter out these waste products. A large number of small blood vessels (capillaries) are an essential component of this filter. After 20-30 years, they start to leak and useful protein is lost in the urine.

It was stated that interruption of the renin-angiotensin system slows the progression of renal diseases in patients with type 1 diabetes, but similar data are not available for patients with type 2 .

\section{Diabetic Cardiomyopathy}

Patients with both diabetes and ischemic heart disease seem to have an enhanced myocardial dysfunction leading to accelerated heart failure (diabetic cardiomyopathy). Thus, patients with diabetes are prone to congestive heart failure.

\section{Diabetic Neuropathy}

Diabetic neuropathy results in a gradual loss of nerve function which limits the amount of sensation on the plantar aspects of the feet. This diminished sensation disables individuals from being able to feel the onset 
or occurrence of a foot injury. As a result, patients with this disease are more inclined to experience plantar ulceration.

People with DM can develop nerve problems at any time, but the longer a person has diabetes, the greater the risk. Acharya et al. state that abnormal plantar pressures play a major role in the pathologies of neuropathic ulcers in the diabetic foot.

\section{Diabetic Retinopathy}

Diabetes mellitus often results in diabetic retinopathy which is caused by pathological changes of the blood vessels which nourish the retina. DR is the main cause of new cases of blindness among adults aged 2074 years. During the first 20 years of the disease, nearly all patients with type 1 diabetes and $>60 \%$ of patients with type 2 diabetes have retinopathy. In the Wisconsin Epidemiologic Study of DR, 3.6\% of younger-onset patients (type 1 diabetes) and 1.6\% of older-onset patients (type 2 diabetes) were legally blind. In the youngeronset group, $86 \%$ of blindness was attributable to DR. In the older-onset group, in which other eye diseases were common, one-third of the cases of legal blindness were due to DR.

DR occurs when the increased glucose level in the blood damages the capillaries, which nourish the retina. As a result of this damage, the capillaries leak blood and fluid on the retina. The visual effects of this leakage are features, such as microaneurysms, hemorrhages, exudates, cotton wool spots or venous loops, of DR.

\section{Exudates}

Exudates are accumulations of lipid and protein in the retina. Typically they are bright, reflective, white or cream colored lesions seen on the retina. They indicate increased vessel permeability and an associated risk of retinal edema. Although, not sight threatening in themselves, they are a marker of fluid accumulation in the retina. However, if they appear close to the macula center they are considered sight threatening lesions.

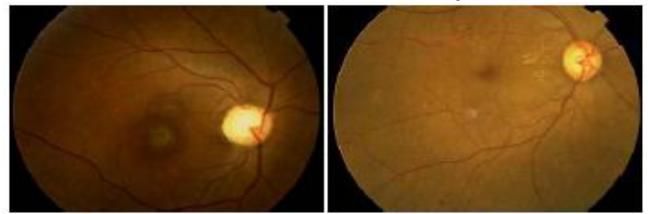

a)

b)
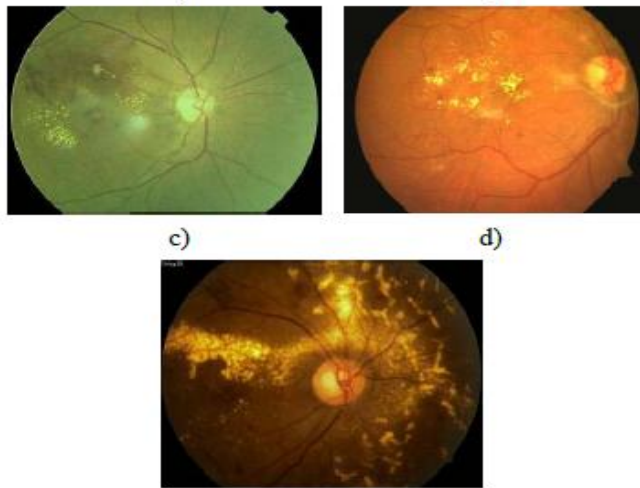

Fig.1 Classified retina images a) Normal b)Mild c)Moderated) Severe e) Very severe

\section{Proposed Method}

Exudate detection is carried out in the following stages.

- Optic Disc Segmentation using Morphological Filtering

- RGB to Gray conversion

- Dynamic Thresholding

- Segmentation Of Exudates and Optic Disc using Morphological Filtering

- Elimination Of Optic Disc using Subtraction

- Detection Of Exudates 


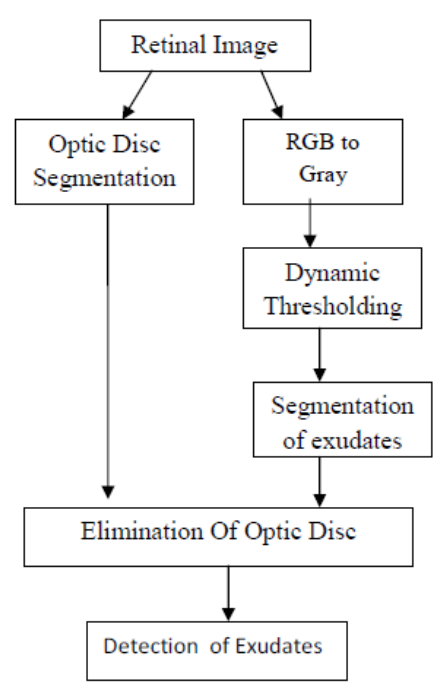

1)Normal Retina Without Exudates

III. Experimental Results

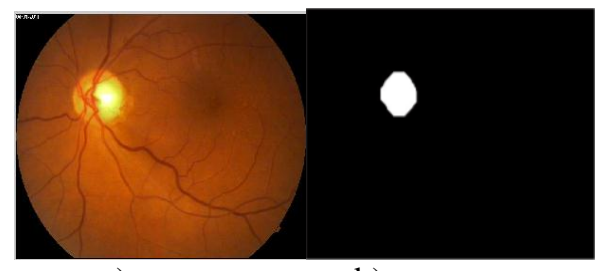

a)

b)

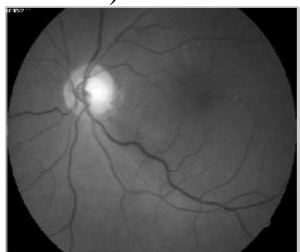

c)

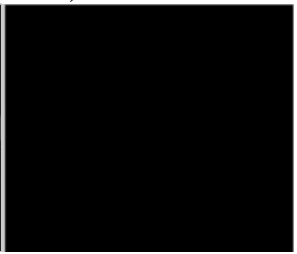

d)

Fig.2 a) Input image b)Optic disc Detection c) Gray image d) No exudates

\section{2) Retina With Exudates}

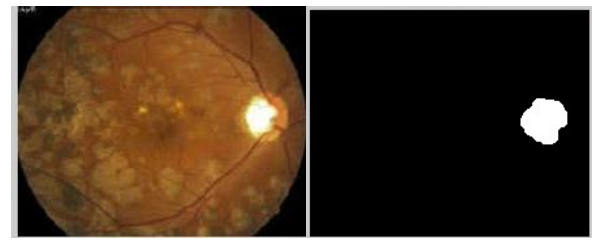

a)

b)

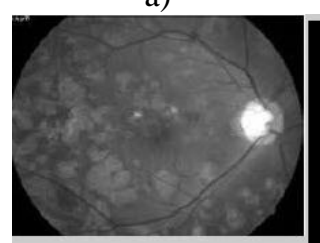

c)

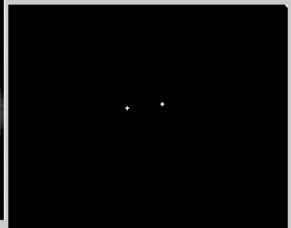

d)

Fig.3 a)Input image b)Optic disc Detection

c) Gray image d) exudates

\section{Conclusion And Future Work}

Diabetes is a metabolic disease that affects tens of millions of people around the world. The statistics shows that this number of diabetic patients will double over the course of future years. Diabetic retinopathy is a sever disease that can multiply leave permanent consequences on the normal function of the human organism, 
one of them being negatively affecting visual function. Regular screenings and medical checkups along with adequate treatment can prevent the further development of the disease.

We proposed cost-effective algorithm for automated detection of exudates and classification of retina images with the goal to assisting ophthalmologist/medical doctor in diagnostics procedures.

The scope of our future research is related to various lesion identification and improved retina image classification based on detecting additional anomalies, like haemorrhages and microaneurysms, caused by diabetes.

\section{References}

[1] Vesna Zeljković, Milena Bojic, Claude Tameze, Ventzeslav Valev "Classification Algorithm of Retina Images of Diabetic Patients Based on Exudates Detection"IEEE, 2012.

[2] Anantha Vidya Sagar, S.Balasubramaniam, V.Chandrasekaran, “A Novel Integrated Approach using Dynamic Thresholding and Edge Detection (IDTED) for Automatic Detection of Exudates in Digital Fundus Retinal Images",IEEE 2007.

[3] Thomas Walter_, Jean-Claude Klein, Pascale Massin, and Ali Erginay, " A Contribution of Image Processing to the Diagnosis of Diabetic Retinopathy-Detection of Exudates in Color Fundus Images of the Human Retina",IEEE2002

[4] Akara Sopharaka, Bunyarit Uyyanonvara, Sarah Barman, " Automatic Exudate Detection for Diabetic retinopathy screening", ScienceAsia 35 (2009)

[5] S. Saheb Basha, Dr. K. Satya Prasad, "Automatic Detection of Hard Exudates in Diabetic retinopathy using Morphological Segmentation and Fuzzy Logic”, IJCSNS International Journal of Computer Science and Network Security, VOL.8 No.12, December 2008

[6] Anitha Somasundaram and Janardhana Prabhu, "Detection of Exudates for the Diagnosis of Diabetic retinopathy" 2013 Innovative Space of Scientific Research Journals

[7] Oliver Faust \& Rajendra Acharya U. \& E. Y. K. Ng . Kwan-Hoong Ng \& Jasjit S. Suri, "Algorithms for the Automated Detection of Diabetic Retinopathy Using Digital Fundus Images: A Review” Springer ,April 2010

[8] Arulmozhivarman Pachiyappan, Undurti N Das, Tatavarti VSP Murthy and Rao Tatavarti "Automated diagnosis of diabetic retinopathy and glaucoma using fundus and OCT images "Lipids in Health and Disease, 2012. 